\title{
Dilthey y el giro antropológico de la filosofía*
}

\section{Dilthey and the anthropological turn of philosophy}

\author{
ANDRZEJ PRZYLEBSKI \\ Universidad de Poznan, (Polonia)
}

Recibido: 01/02/2012 Aceptado: 19/03/2013

\section{RESUMEN}

Este texto es un análisis crítico de la visión del ser humana contenida en la filosofía hermenéutica de la vida elaborada por Dilthey. Dilthey basó su temprana epistemonología de las humanidades en la, así llamada, psicología descriptiva que anticipaba la fenomenología husserliana. Intento mostrar que este concepto está explícitamente conectado con una antropología filosófica que intenta descubrir - sobre la base de una investigación histórica de la cultura humana - las categorías universales que describen la esencia del ser humano. Como consecuencia de elaborar una reflexión sobre la corporalidad de la existencia humana, la filosofía de la vida de Dilthey tiene alguna ventaja sobre la fenomenología de Husserl y la ontología fundamental de Heidegger. La vida es para él algo más que un modo de autorrealización de la conciencia: las formas de vida espirituales y culturales tienen alguna base «im naturgebundenem Leben»

\section{ABSTRACT.}

This text is a critical analysis of the vision of human being contained in Dilthey's hermeneutical philosophy of life. Dilthey based his early epistemology of the humanities on so-called descriptive psychology that anticipated the phenomenology of Husserl. I try to show that this

* This article was written in frame of the scientific grant of NCN 2013/09/B/HS1/00282

(Hermeneutyka. Od sztuki interpretacji o teorii filozofii rozumienia).

(C) Contrastes. Revista Internacional de Filosofia, vol. XXV-Nº3 (2020), pp. 69-79. ISSN: 1136-4076 
concept is explicitly connected with a philosophical anthropology that tries to discover - on a basis of historical research of human culture - the universal categories describing the essence of human being. Because of reflecting on the corporeality of human existence Dilthey's philosophy of life have some advantage over the phenomenology of Husserl and fundamental ontology of Heideggeer. Life is for him something more than a way of self-realization of consciousness: spiritual and cultural forms of life have some basis «im naturgebundenem Leben».

KEY WORDS

DILTHEY; ANTHROPOLOGICAL PHILOSOPHY; HERMENEUTICS

\section{INTRODUCCIÓN}

ELFINAL DE LOS AÑOS 30 del siglo XX estuvo marcado por importantes transformaciones en el ámbito de la filosofía europea. La impresionante aparición de Martin Heidegger el año 1927 con la publicación de su obra maestra, Ser y tiempo, así como los tratados de Cassirer, Jaspers, Scheler y Plessner -por nombrar sólo los más importantes- condujeron a una novedad que cambió esencialmente no sólo la filosofía académica. El propio Heidegger lo interpretaba como un «giro ontológico» de la filosofía, que debía superar la unilateralidad de su orientación hacia el fenómeno del saber -y por tanto, hacia la teoría del conocimiento-, que debía fundamentar las ciencias positivas--. La realidad-el «Dasein»- debía estar de nuevo, como para los griegos, en el centro de la investigación filosófica. Pero como en la obra de Heidegger este giro ontológico se realizó como una ontología fundamental, que comprendía un «análisis existencial», se puede hablar con fundamento, a mi entender, de un «giro haciael ser humano». En ese sentido, el ser humano se concebía como una totalidad concreta, histórica y viva, que gracias al lenguaje crea un mundo en torno a sí. Max Scheler, que marcó la novedad antropológica con su tratado El puesto del hombre en el cosmos, no lo veía de otro modo cuando sostenía que el animal vive en un mundo circundante mientras que el ser humano tiene un mundo.

Llegó así, en la tercera década del pasado siglo, el nacimiento de la antropología filosófica, y no como una subdisciplina de la filosofía, como siempre había sido, sino como una orientación fundamental de la filosofía como tal. Esta aparición, que se puede designar como «giro antropológico de la filosofía», tomó diferentes formas: desde una filosofìa existencial en el sentido de Karl Jaspers, pasando por el análisis del Dasein de Heiddegger y la ya clásica posición de Scheler, hasta la filosofía de la cultura de Cassirer.

1 Tal vez sea significativo señalar que la Sociedad Suiza de Filosofía dedicó a este fenómeno una jornada en mayo de 2012, que llevaba el título «Giro antropológico de la filosofía». 
Hablar de un giro antropológico de la filosofía puede ser un asunto controvertido. En este contexto se habla con frecuencia de un giro «ontológico» (o bien, metafísico), ligado nos sólo al nombre de Heidegger sino también al de N. Hartmann. Está ampliamente establecido el giro «linguístico», que comenzó con Wittgenstein, pero que fue continuado mediante la hermenéutica de Heidegger y Gadamer. ${ }^{2}$ El papel que jugó la hermenéutica de Dilthey en este proceso no estaba, a mi entender, suficientemente aclarado. El propósito del presente texto es, al menos en parte, llenar ese vacío. Éste contiene resultados de una investigación que deseo presentar a un público más amplio, para iniciar un debate acerca de la contribución de Dilthey a este importante giro de la filosofía occidental.

\section{LA FILOSOFÍA HERMENÉUTICA DE LA VIDA DE DILTHEY EN EL CONTEXTO DE LA ÉPOCA}

El paso del siglo XIX al XX estuvo marcado por una confrontación entre las tres principales corrientes de la filosofía de aquel tiempo: el neokantismo, la fenomenología y la filosofía de la vida. La antropología filosófica ha surgido propiamente como consecuencia de la investigación fenomenológica. Con esto me refiero no sólo a pensadores como Max Scheler, sino también a Martin Heidegger, Helmuth Plessner y Roman Ingarden. Esto parece evidente. En el neokantismo, por el contrario, esta transformación apenas tuvo importancia. ${ }^{3}$ La contribución de la filosofía de la vida es aquí mucho más difícil de apreciar, pues en cada uno de los clásicos de esta orientación -me refiero a Bergson, Simmel y Dilthey- incide de manera diferente. En este texto deseo centrarme en la filosofía de la vida de Wilhelm Dilthey, en particular desde el supuesto de que su contribución al giro antropológico de la filosofía, aunque olvidado o mejor dicho no descubierto, parece ser evidente. La tesis principal de este trabajo es ésta: para Dilthey, no de otro modo que para Scheler o Heidegger, en el primer plano de su interés filosófico está el «hombre entero»- es decir, un ser corporal e histórico-espiritual. En las dos partes principales de su teoría, es decir, en la hermenéutica y en la filosofía de la vida, intenta dar razón de este punto de vista.

Quienquiera que se ponga en contacto con la obra de Dilthey, se verá obligado a constatar, de entrada, que no forma una totalidad terminada y perfilada,

2 Es suficiente indicar dos importantes expresiones/sentencias de ambos - «el lenguaje es la casa del ser» (Heidegger) y «el ser que puede ser comprendido es el lenguaje».

3 Para entender esto es suficiente recordar los títulos de las principales obras de Windelband, Cohen, Natorp, Rickert o Lask. Es este caso se puede hablar únicamente de un «giro ontológico» del neokantismo de Baden, que es manifiesto en la obra tardía de Rickert. 
sino que manifiesta un proceso de pensamiento no concluido que únicamente de un «giro ontológico» del neokantismo de Baden, que es manifiesto en la obra tardía de Rickert, fue interrumpido en 1911 por su muerte inesperada; y, en segundo lugar, que se inspira en tres grandes tradiciones y acomete el intento de integrarlas con todo el empeño de que es capaz. Con estas tres grandes tradiciones me refiero a la filosofía transcendental de Kant, que Dilthey, en confrontación con los neokantianos, quería completar con una cuarta crítica, una «crítica de la razón histórica»; el idealismo absoluto de Hegel, del que tomó la idea de «espíritu objetivo» y que desarrolló ampliamente en su filosofía; y, en tercer lugar, la «filosofía de la voluntad» iniciada en las obras de Schopenhauer y Nietzsche, que configuró como una «filosofía de la vida» sistemática.

Dilthey vinculó con claridad las tareas de la filosofía con la comprensión de todo lo que pertenece al ámbito de lo espiritual. La naturaleza exterior a nosotros le interesaba poco, la consideraba como algo extraño e incomprensible para el ser humano. Podemos, desde luego, explicarla con ayuda de construcciones mentales acertadas, para dominarla hasta cierto punto, pero nunca podemos abarcarla (mejor dicho, comprenderla), a diferencia de la cultura y la historia, que es la esfera de la libertad y creatividad humana, y que es accesible por ello a nuestra comprensión. La cultura es para Dilthey la articulación de la vida, dicho más precisamente: de la vida humana. Es objeto de investigación de muchas ciencias que la filosofía respalda -Dilthey insistía en caracterizarlas como ciencias del espíritu y no como ciencias de la cultura o ciencias humanas. El papel de la filosofía es, por tanto, especialmente interesante, pues pretende descubrir e interpretar conceptualmente los fundamentos de la construcción humana de la cultura a partir de los resultados de las ciencias particulares. Es muy significativo el título de la última obra principal de Dilthey, aparecido póstumamente - La estructuración del mundo histórico en las ciencias del espiritu.

Desarrolló su propósito de fundamentar filosóficamente las ciencias del espíritu, de cimentarlas epistemológicamente -y, por tanto, de llevar a cabo la anunciada crítica de la razón histórica- de forma completamente diferente a los neokantianos de la llamada escuela de Baden. Como Schopenhauer o Nietzsche, no creía en la fuerza todopoderosa de la razón. Admitió el carácter irracional, que excede toda razón, de la realidad concebida como vida. El concepto principal y el punto de referencia capital no fue para él la razón sino la vida. Y aunque en ocasiones habló de la vida como una totalidad orgánica, es evidente que su filosofía se refiere a la vida humana y se orienta a ella. Pues lo que interesa a la filosofía es la vida humana que manifiesta sus formas en la historia.

Su distanciamiento de la filosofía transcendental se aprecia con claridad en que se aleja inequívocamente del sujeto transcendental. El conocimiento acontece, original y fundamentalmente, al servicio de la vida, en primer lugar 
de la supervivencia y después del crecimiento de la vida, y siempre está históricamente determinado, inserto en un contexto. Para Dilthey la «subjetividad transcendental» o la «conciencia pura» como fundamento del conocimiento, no tiene base histórica ni real. Es universalmente conocida la fórmula de Dilthey, en la que afirma que por las venas del sujeto kantiano no circula sangre verdadera, sino una delgada savia de la «razón como mera actividad intelectual». Ese punto de partida tenía que ser abandonado. Por eso pone en su lugar un hombre histórico, un hombre de carne y hueso, con todas sus vivencias (Erlebnisse), pasiones, ideales, valoraciones y objetivos, condicionados individual, social y temporalmente. Y todo ello, unido a una perspectiva de conocimiento.

Esta actitud está ya visiblemente en su primera gran obra, Introducción a las ciencias del espíritu, de 1883. En este escrito es manifiesta también su posición con respecto a la antropología, cosa que aquí nos interesa especialmente. La antropología aparece ahí en el contexto de una ciencia fundamental que Dilthey postula. Es una psicología analítico-descriptiva -completada más tarde con una psicología comparada- que debe servir como fundamento teórico para el conocimiento de lo que los hombres han producido. La prioridad de la psicología es claramente comprensible: si se parte del «principio de fenomenalidad», que establece que la realidad está dada para nosotros en la vivencia (en vivencias, en fenómenos), entonces un análisis de las vivencias (mejor dicho, de los actos de la conciencia) debe gozar del primado teorético. La ciencia propuesta por Dilthey como alternativa a la psicología empírica puede ser considerada, a mi entender, como una anticipación de la primera fenomenología de Husserl y Scheler.

Una psicología así concebida vincula a Dilthey con la antropología. En una conferencia pronunciada en 1863 sobre la lógica y el sistema de la ciencia afirma incluso que la psicología y la antropología constituyen la ciencia fundamental del espíritu. ${ }^{5}$ Encontramos formulaciones similares en la obra principal de 1883, cuando Dilthey señala que «así consideradas, la antropología y la psicología constituyen la base de todo el conocimiento de la vida histórica, lo mismo que de todas las reglas para la marcha y dirección de la sociedad». ${ }^{6}$ Es interesante además que en esta frase se utiliza la palabra «es»y no «son», lo que parece

4 Cfr. W. Dilthey, Gesammelte Schriften (en adelante: GS), vol. I, Stuttgart/Götingen 1979 , p. XVII.

5 El título completo reza así: «Esquema de la lógica y el sistema de las ciencias filosóficas para clases» (cito según el texto de Hans Ineichen con el título «Wilhelm Dilthey. Fundamentos lógicos y antropológicos de las ciencias del espíritu», en: F. Decher y J. Hennigfeld (eds.), Antropología filosófica en el siglo XIX, Würzburg 1992, p. 171.

6 W. Dilthey., GS I, p. 28. H. Ineichen, Op.cit.., p. 171. [Traducido literalmente: «la psicología y la antropología es el fundamento...». Nota del T.] 
indicar que las dos disciplinas son propiamente una y la misma. No obstante, si se considera su segunda obra principal, la cosa parece complicarse un poco.

En La estructuración del mundo histórico en las ciencias del espíritu escribe que no puede haber ciencia alguna que sea capaz de abarcar el reino de la vida espiritual del hombre. La más próxima a ello es para él la antropología que, por medio de la penetración en las relaciones concretas de las manifestaciones culturales y en las estructuras profundas de la vida espiritual de un individuo, puede establecer fórmulas, mejor dicho, principios, que se ajustan perfectamente al carácter general de una ciencia caracterizada de ese modo. ${ }^{7}$ En este sentido, la antropología sería una generalización de los resultados de la investigación de la psicología analítico-descriptiva, es decir, de la teoría de la conciencia por una parte y de la doctrina sobre los realizaciones del espíritu objetivo por otra.

\section{CONCEPCIÓN DILTHEYANA DE LA ANTROPOLOGÍA}

El modelo antropológico que Dilthey adopta se basa en el supuesto de que el hombre es un ser biológico que quiere sobrevivir en un entorno. Este entorno es tanto de carácter natural como social. La frase «el mundo del hombre es menos un mundo natural que un mundo social» muestra dónde pone el acento. Para alcanzar este objetivo al hombre no le basta sólo conocer su entorno, tiene que sentirlo, captar sus estímulos, y tiene que actuar en él para alcanzar sus fines. En comparación con otros seres vivos, el ser del hombre se caracteriza por que en él coexisten e interactúan tres clases de vivencias que están unidas a tres tipos de actitudes respecto de la realidad. La actitud más importante para Dilthey no es la cognitiva, sino la emocional: responder a los efectos del entorno, en primer lugar, con sentimientos de sufrimiento/dolor y/o de placer/ gozo. Éste es el «lugar de nacimiento» de las valoraciones y los valores.

Así concebidos, los sentimientos que surgen del contacto directo con la realidad vinculan al hombre con un «ahora» de su existencia, con el momento presente. Por otra parte, nos muestran también qué deseamos, en la consecución de qué podemos empeñarnos, en qué podemos esforzarnos para «llevarlo-alser». Esto lleva consigo la necesidad de la acción y por tanto el establecimiento de fines, y de este modo se abre para nosotros la dimensión de lo futuro y de lo querido.

Se puede decir, no sin fundamento, que las dos actitudes mencionadas hacia la realidad están presentes también en los animales superiores: sienten y se relacionan con ella. Incluso si se afirma esto, la diferencia con el hombre consistiría en que a los animales les falta la tercera dimensión, que se da sólo en el hombre con el pensamiento abstracto y mediado simbólicamente. Como

7 W. Dilthey, Aufbau der geschichtlichen Welt in den Geisteswissenschaften, GS, vol. VII, p. 331. 
señaló claramente Ernst Cassirer, en el hombre la reacción a los estímulos está separada por una pausa de pensamiento que hace posible y necesario el pensamiento simbólico. ${ }^{8}$ Dilthey designa esta actitud como «actitud objetiva»: el conocimiento humano forma objetos que pueden ser discutidos intersubjetivamente, es decir, con independencia de las experiencias individuales e irrepetibles con una cosa. De este modo nace un saber objetivo. Pero es siempre, no debe olvidarse, un conocimiento de lo que ha ocurrido, por tanto apunta a la dirección de pasado. Esta actitud, que tras muchos siglos de evolución humana desemboca en la ciencia moderna, da al hombre una increíble supremacía frente a todos los animales. Pero no por ello deja de estar anclada en las otras dos actitudes, es decir, profundamente unida a ellas.

Las tres dimensiones antes mencionadas determinan una estructura fundamental común a todos los hombres, se podría decir, antropológica. Esta estructura puede ser llenada en cada hombre con distintos contenidos y distintas intensidades, lo que permite explicar las diferencias entre los individuos. Es en las acentuaciones como el hombre de acción se diferencia del hombre de investigación o del que se dedica a la contemplación del ser, mejor dicho, del Dasein. Apenas sería posible imaginar un ser humano en que faltara por completo una de estas dimensiones vitales. Esta estructura común de la vida espiritual se concreta en cada caso en lo que Dilthey caracteriza como «conexión adquirida de la vida psíquica» y que -al menos en parte- es el resultado del crecimiento del individuo en una comunidad cultural.

Sentir, querer y conocer - estos tres elementos fundamentales de la estructura de nuestro ser, reemplazan en Dilthey lo que antes se ponía en el concepto de alma. ${ }^{9} \mathrm{Su}$ conexión tanto temporal como lógica permite explicar, por una parte, la interacción de las «facultades del alma», que antes se consideraban independientes una de otra. Por otra parte, constituye un estado antropológico fundamental, un punto de partida para la comprensión de los procesos histórico-culturales. También es importante señalar aquí que, mediante la temporalización de las tres actitudes, Dilthey anticipa la doctrina de Heidegger sobre la «maduración del Dasein». ${ }^{10}$ Es también claro que Dilthey va más allá de Heidegger, pues en su antropología tiene en consideración el hombre entero, también la dimensión de la corporalidad que falta en Heideger. El hombre de Dilthey es un ser que experimenta el mundo. En la medida en que acepta como punto de partida la experiencia vital -y no la experiencia de los fenómenos

8 Cfr. E. Cassirer, An Essay on Man. An Introduction to a Philosophy of Human Culture, New York 1945, cap. II y III.

9 También sostiene esta opinión el importante investigador de Heidegger Hans Ineichen(cfr. Op. cit., p. 174).

10 Cfr. además: M. Heidegger, Sein und Zeit, Tübingen 1979 (15. ed.), p. 61-71. 
naturales, la experiencia natural, como Kant-, hace secundaria la relación sujeto-objeto. Esto lleva a la eliminación de un catálogo cerrado de categorías a priori, que deberían determinar el conocimiento humano - y por tanto la experiencia. Dilthey es deliberadamente anti-apriorista: su concepción de la captación humana de la realidad le sitúa en la cercanía de Friedrich Nietzsche. Esas categorías son para él cristalizaciones rígidas, mejor dicho, petrificadas, de la confrontación cognoscitiva con el mundo, que los individuos y grupos almacenan, pero que también a veces olvidan. Por eso, en lugar de una tabla de categorías apriorística, Dilthey propone la aceptación de categorías que hagan justicia al origen y al alcance de las formas de pensamiento nacidas en la historia. Menciona una serie de tales categorías (como: sentido, valor, fin, ideal), y advierte que éstas forman un catálogo abierto, pues la vida no se deja encerrar en un corsé conceptual.

Es especialmente destacable que esta antropología así concebida puede fundamentar la posibilidad de comprensión sobre-cultural, o mejor dicho, intercultural de los hombres. Con ella podemos comprender culturas extrañas, porque están formadas por hombres dotados de estructuras psíquicas similares; aunque esta comprensión nunca pueda ser completa. Esto nos proporciona el primer punto de partida, que afirma que nos las habemos con un ser que siente, quiere y piensa. Esto a su vez constituye la condición de posibilidad de una hermenéutica intercultural que puede oponerse a un relativismo total, una hermenéutica que se basa en la idea de la unidad de la razón humana, aunque esa unidad es concebida no como algo dado, sino como algo que se alcanza a través de un proceso. Esto significa: una razón histórica. ${ }^{11}$

\section{CONCLUSIÓN}

Nos acercamos al final de esta breve reflexión. Todo lo que he expuesto lleva a la conclusión, según espero, de que Dilthey no sólo fue uno de los más importantes teóricos de las ciencias del espíritu y cofundador de la filosofía hermenéutica, sino que también contribuyó a que se produjera el giro antropológico de la filosofía. ${ }^{12} \mathrm{La}$ fractura del marco especulativo de la concepción hegeliana de la historia como historia de la razón absoluta lleva a Dilthey a concebir la historia como un proceso abierto, que se forma mediante las acciones espontáneas y libres de los individuos. La vida humana se realiza en la forma de configuraciones culturales históricamente cambiantes. La variedad

11 Gadamer ha introducido la idea de una razón hermenéutica, para ponerla frente a la «razón absoluta» de Hegel. Cfr. mi artículo: A. Przylebski, «Rozum hermeneutyczny», in: Z. Drozdowicz (ed.), Racjonalność w nauce i w życiu społecznym, Poznań 2009, p. 39-53.

12 También sostiene esta opinión Gerhard Arlt en su libro Philosophische Anthropologie, Stuttgart 2001, p. 29. 
de las culturas lleva consigo una pluralidad de tipos de racionalidad. Dilthey intenta, como es sabido, compaginar esta circunstancia con la tipología delas concepciones del mundo. ${ }^{13}$ A sus ojos el hombre es tanto sujeto como objeto de la historia. Pienso que en su filosofía - si se la considera como una unidad-se puede encontrar una dialéctica entre la filosofía de la vida y la hermenéutica. La vida se articula, las articulaciones de la vida se comprenden, su comprensión condiciona la vida vivida -la cual, por tanto, ya no queda como no-reflexiva y produce nuevas nuevas articulaciones vitales más ricas. De este modo, la vida no se reduce a una corriente de vivencias, a algo que transcurre y que es irracional, sino que se convierte en un medio, que garantiza una cierta estabilidad y continuidad. ${ }^{14}$

La posición de la «vida-que-se-vive-a-sí-misma», que Dilthey designa con el término «autognosis», ofrece a su filosofía perspectivas completamente nuevas. El centro viene a ser, por eso, el ámbito de la vida experimentada, y eso quiere decir: de la propia praxis vital. Esto significa una rehabilitación de la vida y de la experiencia precientífica del mundo. Este punto de vista -casi revolucionario para aquel tiempo-no está sin embargo libre de problemas que, como conclusión, quisiera al menos señalar. En relación con la fundamentación de las ciencias del espíritu, Hans Ineichen ha visto, no sin razón, el peligro de una contradicción entre lo puramente lógico y lo antropológico-psicológico. Reprocha a Dilthey la confusión o la mezcla del origen con la valoración. Al tiempo que acepta por completo la orientación de la teoría del conocimiento de Dilthey, Ineichen intenta minimizar este peligro apuntando al concepto de intencionalidad, forjado en la fenomenologia, que podría ser una especie de puente entre ambos órdenes. La intencionalidad tiene siempre una faceta subjetiva (se refiere a un pensamiento de alguien) y una faceta objetiva (concierne a un objeto pensado, es un «pensar-sobre-algo»" ${ }^{15}$. Esto podría apuntar a una solución del problema. Pero, pienso, también habría que dar la razón a Gerhard Arlt cuando dice: la indecisión de Dilthey entre una fundamentación epistemológica (questio iuris) y una fundamentación antropológica (questio facti) resultó ser históricamente muy fecunda. ${ }^{16}$ Abrió el camino al rapidísimo desarrollo de la antropología filosófica, a pesar de las protestas de un Husserl o un Heidegger.

13 Cfr. la teoría de las concepciones del mundo de Dilthey, GS, vol. VIII, Stuttgart/ Göttingen 1977.

14 Este aspecto se expone claramente en dos libros de Ferdinand Fellmann Lebensphilosophie. Elemente einer Theorie der Selbsterfahrung, Reinbek b. Hamburg 1993 y Hermeneutik nach Dilthey, Reinbek b. Hamburg 1991.

15 Cfr. H. Ineichen, Op cit., p. 177-178.

16 Cfr. G. Arlt, Op.cit., p. 38. 
Otra dificultad de la fundamentación antropológica de las ciencias del espíritu por parte de Dilthey consistiría en que se le puede reprochar una cierta forma de apriorismo. En la medida en que Dilthey alude a una estructura profunda del ser humano, parece referirse al problema del ser humano. Las tres dimensiones -sensitiva, volitiva y cognoscitiva- serían una especie de jaula ahistórica, cosa que estaría en contradicción con su declarado historicismo. Su frase probablemente más famosa reza así: lo que el ser humano es, se lo dice sólo la historia. Dilthey podría hacer frente a esta objeción de dos formas. En primer lugar, podría decir que ésa es solamente una división provisional, por tanto: científica, de una unidad originaria de vida. No se descarta que haya o pueda haber en el futuro otras posibles divisiones de dicha unidad. Y en segundo lugar, podría responder: mi concepto sólo perderá valor cuando podamos imaginar un ser humano del futuro, en el que falte por completo alguna de estas tres dimensiones. Parece, no obatante, que en la medida en que el ser humano sea corporal y mortal, y esto significa: caracterizado por exigencias biológicas, por la lucha por la vida y por la configuración de la vida, en esa medida la conexión efectiva de los tres elementos será indispensable. Pues sólo ella puede garantizar algún éxito.

Se puede aceptar con fundamento que en los años 30 del siglo pasado tuvo lugar un «giro antropológico de la filosofìa». Éste no fue llevado a cabo solamente por los tratados de los tres clásicos de la antropología filosófica (Scheler, Plessner, Gehlen), sino que se produjo por una serie de pensadores que no tenían como emblema la palabra «Antropología» (entre otros, Cassirer, Jaspers, Heidegger). La filosofía antropológica fue también una expresión del cambio tranquilo de la filosofía europea al comienzo del siglo XX, aunque su ímpetu, de forma explícita, decayó con el tiempo. Tras la segunda guerra mundial, este giro se continuó en el marco de la filosofía hermenéutica, de la filosofía de la cultura y de la filosofía del lenguaje. La filosofía hermenéutica de la vida de Dilthey fue, sin ninguna duda, una parte de este proceso. Y aunque sus escritos sólo contenían una «antropología mínima», acarrearon un cambio radical, en el marco del cual el hombre concreto, de carne y hueso e histórico, en todas sus facetas -y no sólo el mero ser o el puro conocer -se convirtió en el objeto principal de la filosofía. Es un problema de esta antropología mínima el hecho de que resulta de una extraña unión del historicismo («lo que es el hombre, sólo se lo dice la historia») y del apriorismo (la temporalidad es la esencia del hombre), unión que precisa ser aclarada. Pertenece a su avance, a mi juicio, el establecimiento del fundamento de una hermenéutica intercultural que, lejos de la presunción eurocéntrica y del relativismo posmoderno, que iguala todas las culturas, puede contribuir a una mejor comprensión del ser humano en y por medio de la cultura. No hay que olvidar, además, que Dilthey es el descrubridor del «largo camino» propuesto por Paul Ricoeur-frente al 
análisis existencial de Heidegger-, un camino a través la filosofía de la cultura y de las ciencias de la cultura.

ANDrzej PrzylebsKi es catedrático de filosofía en la Universidad de Poznan (Polonia).

\section{Publicaciones recientes}

Sense, Meaning and Understanding. Towards a Systematic Hermeneutical Philosophy, LIT Verlag Berlin 2013 (my book in English, issued 2013 in Germany);

Hermeneutische Philosophie der Wissenschaft. Bemerkungen im Ausgang von Gadamer und Fleck, University Press, Rostock 2011.

Dirección electrónica: andrzej@przylebski.com 
